\title{
A VALIDATED STABILITY INDICATING RP-HPLC METHOD FOR ESTIMATION OF PSEUDOEPHEDRINE, AMBROXOL AND DESLORATIDINE IN BULK AND PHARMACEUTICAL DOSAGE FORM
}

\author{
Sreedhar Lade ${ }^{*}$ and Y. Rajendra Prasad ${ }^{2}$ \\ ${ }^{* 1}$ College of Pharmaceutical Sciences, Acharya Nagarjuna University, \\ Nagarjuna Nagar, Guntur-522510, A.P., India. \\ 2University College of Pharmaceutical Sciences, Andhra University, \\ Visakhapatnam-530003, A.P., India. \\ *Corresponding author e-mail: sreedharlade7@gmail.com
}

\begin{abstract}
:
A new simple, precise, selective, accurate and rapid reverse phase high performance liquid chromatographic stability indicating method had been developed and validated for simultaneous quantitative determination of Pseudoephedrine, Ambroxol and Desloratidine in bulk and pharmaceutical dosage form. The chromatographic separation was achieved with InertisilODS 3V, $(250 \times 4.6 \mathrm{~mm})$ and $5 \mu \mathrm{m}$ particle size column. The optimized mobile phase consisting of phosphate buffer: Acetonitrile: Methanol (50:20:30 $\% \mathrm{v} / \mathrm{v}$ ). The flow rate was $1.0 \mathrm{~mL} / \mathrm{min}$ and eluents were detected at $225 \mathrm{~nm}$ using PDA detector. The retention time of Pseudoephedrine, Ambroxol and Desloratidine were found to be 2.379, 3.971 and 5.450 respectively. The percentage recoveries for three molecules were found to be in the range of $99-102 \%$. The calibration curve was constructed between peak area vs concentration and demonstrated good linearity in the range of $2.5-15 \mu \mathrm{g} / \mathrm{ml}$ for Pseudoephedrine, $30-180 \mu \mathrm{g} / \mathrm{ml}$ for Ambroxol and $2.5-15 \mu \mathrm{g} / \mathrm{ml}$ for Desloratidine. Degradation studies were studied for Pseudoephedrine, Ambroxol and Desloratidine under various stress conditions such as acid hydrolysis, base hydrolysis, oxidation, thermal, photochemical and UV. All the degradation peaks were resolved effectively using developed method with different retention times. The developed method was validated according to ICH Q2-R1 guidelines. As the method could effectively separates the degradation products from active ingredients, it can be used for routine analysis of drug both in bulk and pharmaceutical dosage form.
\end{abstract}

Key words: Pseudoephedrine, Ambroxol, Desloratidine, Acetonitrile, Methanol, Buffer, RP-HPLC.

\section{INTRODUCTION:}

Pseudoephedrine $\mathrm{HCl}(\mathrm{PSH})$ is $(1 S, 2 \mathrm{~S})-2-$ (methylamino)-1-phenylpropan-1-ol hydrochloride, it is a stereoisomer of ephedrine and has similar action. PSH and its salts are orally used for the symptomatic relief of nasal congestion and are commonly used in combination with other ingredients in preparations intended for the relief of cough and cold symptoms.[1] PSH as the function of constringing the blood vessel, eliminating mucous membrane congesting and tumefying of nasopharynx, alleviating symptom of the nasal congestion. ${ }^{[2]}$ Only few methods have been described in the literature for the determination of Pseudoephedrine in combination with other drugs by HPLC.[1-2] The chemical structure of Pseudoephedrine $\mathrm{HCl}$ is given in Fig. 1.

\section{How to cite this article:}

Sreedhar Lade and Y. Rajendra Prasad. A validated stability indicating RP-HPLC method for estimation of pseudoephedrine, ambroxol and desloratidine in bulk and pharmaceutical dosage form. Int. J. Adv. Pharm. Biotech., 2015; 1(1): 1-19. 
<smiles>CN[C@H](C)[C@H](O)c1ccccc1</smiles>

Fig. 1: Pseudoephedrine HCl

Ambroxol hydrochloride (AB) [Trans-4-[(2amino-3, 5-dibromobenzyl) amino] cyclohexanol hydrochloride] is a semisynthetic derivative of vasicine obtained from Indian shrub Adhatoda vasica. It is a metabolic product of bromhexine and possesses mucokinetic (improvement in mucus transport) and secretolytic (liquifies secretions) properties. It promotes the removal of tenacious secretions in the respiratory tract and reduces mucus stasis (arresting the secretion of mucus). [3] It is a secretolytic agent used in the treatment of tracheobronchitis, emphysema with bronchitis pneumoconiosis, chronic inflammatory pulmonary conditions, bronchiectasis, bronchitis with bronchospasm asthma. [4-7] It is official in Indian Pharmacopoeia (IP) and British Pharmacopoeia (BP). IP describes High Performance Liquid Chromatography (HPLC) [8] method and BP describes HPLC [9], Spectrophotometric and High Performance Thin Layer Chromatography (HPTLC) methods. Literature survey also reveals HPLC [10-13], Spectrophotometric [14] and HPTLC methods for determination of Ambroxol with other drugs. The chemical structure of Ambroxol is given in Fig. 2.

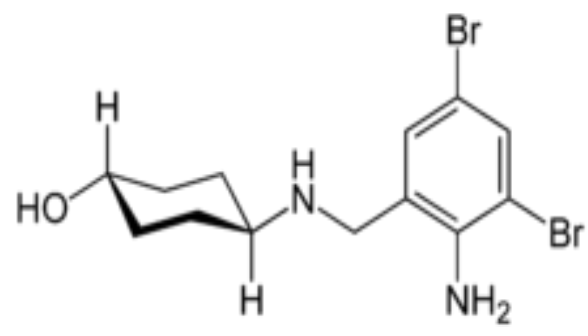

Fig. 2: Ambroxol

Desloratadine (DES) is chemically 8-chloro-6, 11-dihydro-11-(4-piperdinylidene)- $5 \mathrm{H}$ benzo $[5,6]$ cyclohepta $[1,2-b]$ pyridine is a second generation antihistaminic drug.
Desloratadine is an orally administered non sedative, long acting antihistaminic with selective H1-receptor antagonistic activity. Desloratadine is an active metabolite of loratadine. It is used for the relief of symptoms of seasonal allergic rhinitis, perennial (non-seasonal) allergic rhinitis and for the symptomatic treatment of pruritus and urticaria (hives) associated with chronic idiopathic urticarial. [5] Desloratadine is not official in any Pharmacopoeia [5]. Literature survey reveals that RP-HPLC [10, 11, 15, 16, 17] methods for determination of DES individually and in combination with other drugs. Literature survey also reveals spectrophotometric methods [18] for the determination of DES. It is available in the Merck index [19] and Martindale [20], the complete drug reference. The chemical structure of Desloratadine is given in Fig. 3.

Therefore, the main aim of the present work is to develop and validate RP HPLC method for simultaneous estimation of Pseudoephedrine, Ambroxol and Desloratadine in bulk and pharmaceutical dosage forms according to ICH Q2-R1 guidelines. [21] Hence, it has driven the authors to develop a method which is new, simple, precise, and accurate for the simultaneous determination of all the three drugs in their pharmaceutical dosage forms.

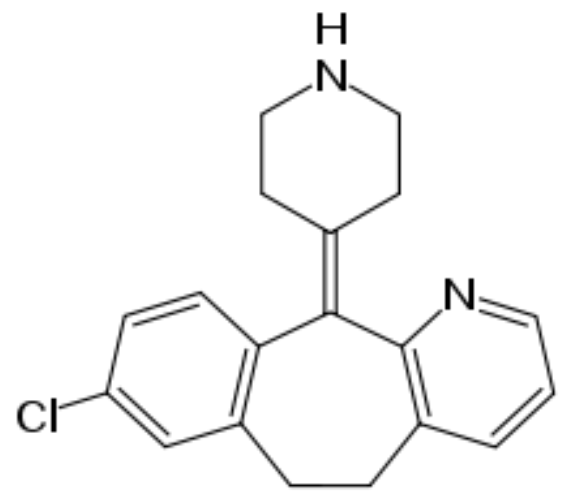

Fig. 3: Desloratadine

The present RP-HPLC method was validated following ICH Q2-R1 guidelines. [21]

\section{MATERIALS AND METHOD:} Chemicals and solvents:

Pseudoephedrine, Ambroxol and Desloratidine were obtained as gift sample from Spectrum Pharma Research Solutions, 
Hyderabad, India. The commercial Pharmaceutical Preparation NUCOPE-AD tablets containing $30 \mathrm{mg}, 60 \mathrm{mg}$ and $5 \mathrm{mg}$ of Pseudoephedrine, Ambroxol and Desloratidine respectively (Marketed by Mankind Pharmaceuticals Private Limited) were procured from local pharmacy. Acetonitrile, Methanol and water used are of HPLC grade.

\section{Instrumentation:}

The chromatographic separations were performed using HPLC-Waters alliance (Model-2695) consisting of an in-built auto sampler, a column oven and 2996 PDA detector. The data was acquired through Empower-2-software. The column used was Inertisil ODS $3 \mathrm{~V},(250 \times 4.6 \mathrm{~mm}$ i.d, $5 \mu \mathrm{m}$ particle size). Meltronics sonicator was used for enhancing dissolution of the compounds.
Elico $\mathrm{pH}$ meter was used for adjusting the $\mathrm{pH}$ of buffer solution. All weighing was done on Sartorius balance (Model AE-160).

\section{Chromatographic conditions:}

The mobile phase consists of Buffer: Acetonitrile: Methanol in the ratio of 50:20:30 $\% \mathrm{v} / \mathrm{v}$. The mobile phase was pumped from solvent reservoir in the ratio of 50:20:30 $\% \mathrm{v} / \mathrm{v}$ to the column in the flow rate of 1.0 $\mathrm{ml} / \mathrm{min}$ whereas run time set was $9 \mathrm{~min}$. The separation was performed on Inertisil ODS 3V column and the column was maintained the temperature of $30^{\circ} \mathrm{C}$ and the volume of each injection was $10 \mu \mathrm{L}$, prior to injection, the column was equilibrated for at least $30 \mathrm{~min}$ with mobile phase flowing through the system. The eluents were monitored at 225 nm. The optimized chromatographic conditions were shown in Table- 1 .

\section{Table-1: Optimized Chromatographic conditions for proposed HPLC method for Pseudoephedrine, Ambroxol and Desloratidine}

\begin{tabular}{|c|c|c|}
\hline S.No. & Parameter & Chromatographic conditions \\
\hline 1. & Instrument & : Waters 2695, High performance Liquid Chromatography \\
\hline 2. & Flow rate & : $1 \mathrm{ml} / \mathrm{min}$ \\
\hline 3. & Column & : Inertisil ODS3V, $250 \times 4.6 \mathrm{~mm}, 5 \mu$ \\
\hline 4. & Detector wave length & : $225 \mathrm{~nm}$ \\
\hline 5. & Column temperature & : $30^{\circ} \mathrm{C}$ \\
\hline 6. & Injection volume & : $10 \mathrm{~L}$ \\
\hline 7. & Run time & : $9 \mathrm{~min}$ \\
\hline 8. & Diluent & : Water: Methanol (30:70) \\
\hline 9. & Mode of separation & : Isocratic mode \\
\hline
\end{tabular}

\section{Preparation of buffer solution:}

Accurately weighed 1.36 gm of Potassium dihyrogen ortho phosphate in a $1000 \mathrm{ml}$ of Volumetric flask to this about $900 \mathrm{ml}$ of milli-Q water added and degassed to sonicate and finally made up the volume with water and adjusted the $\mathrm{pH}$ to 3.0 with dilute ortho phosphoric acid.

\section{Preparation of mobile phase:}

Buffer, Acetonitrile and Methanol were taken in the ratio of 50:20:30. Filtered through 0.45 $\mu$ filter under vacuum filtration.

\section{Preparation of standard solution:}

Accurately weighed and transferred $6 \mathrm{mg}$ of Pseudoephedrine, $5 \mathrm{mg}$ of Desloratidine and
$12 \mathrm{mg}$ of Ambroxol working standards into $10 \mathrm{ml}, 50 \mathrm{ml}$ and $10 \mathrm{ml}$ clean dry volumetric flasks respectively, to this added $7 \mathrm{ml}$ of diluent, sonicated for 30 minutes and finally made up the volume up to the mark with diluents. From the above stock solution, $1 \mathrm{ml}$ was pipette out in to a $10 \mathrm{ml}$ volumetric flask and then make up to the final volume with diluent. $(60 \mu \mathrm{g} / \mathrm{ml}$ Pseudoephedrine, 120 $\mu \mathrm{g} / \mathrm{ml}$ Ambroxol and $10 \mu \mathrm{g} / \mathrm{ml}$ of Desloratidine)

\section{Preparation of sample solution:}

5 tablets were weighed and calculated the average weight of each tablet, then the weight equivalent to 1 tablet was transferred into a $50 \mathrm{ml}$ volumetric flask, $30 \mathrm{ml}$ of diluent was 
added and sonicated for $30 \mathrm{~min}$, further volume made up to the mark with diluent and filtered.

From the filtered solution $1 \mathrm{ml}$ was pipetted out into a $10 \mathrm{ml}$ volumetric flask and volume made up to $10 \mathrm{ml}$ with diluent. $(60 \mu \mathrm{g} / \mathrm{ml}$
Pseudoephedrine, $120 \mu \mathrm{g} / \mathrm{ml}$ Ambroxol and $10 \mu \mathrm{g} / \mathrm{ml}$ of Desloratidine)

Label Claim: $30 \mathrm{mg}$ of Pseudoephedrine +60 $\mathrm{mg}$ of Ambroxol+ $5 \mathrm{mg}$ of Desloratidine HPLC Chromatograms of Standard, Sample and Placebo were shown in Fig. 4-6.

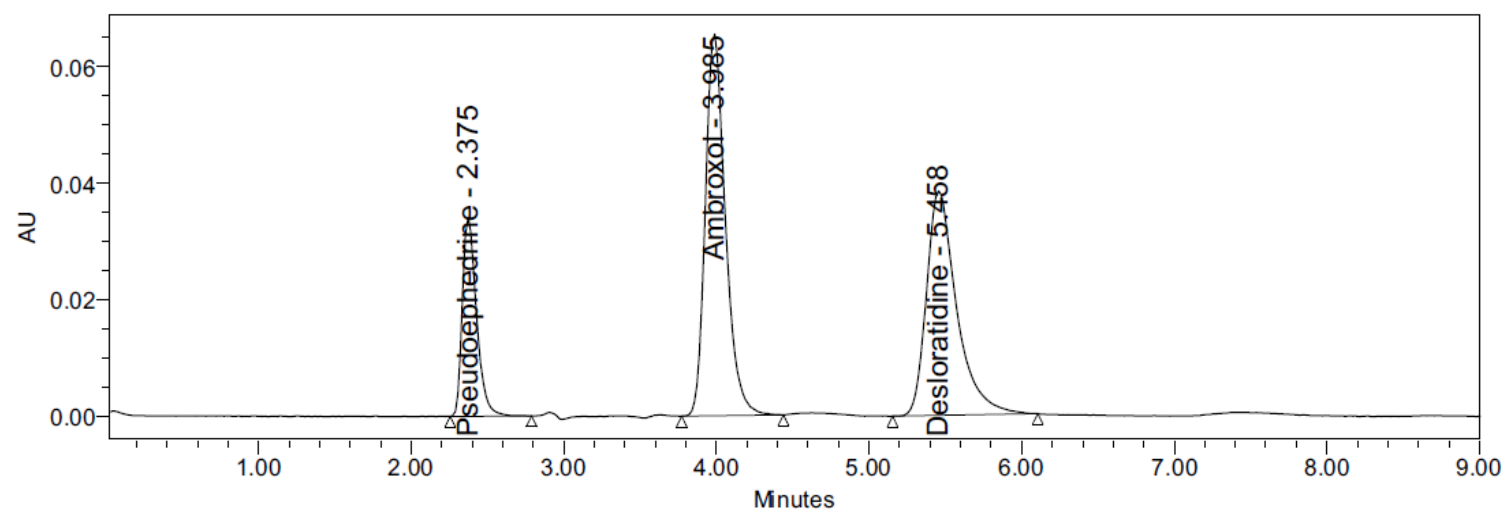

Fig. 4: HPLC Chromatogram of Standard

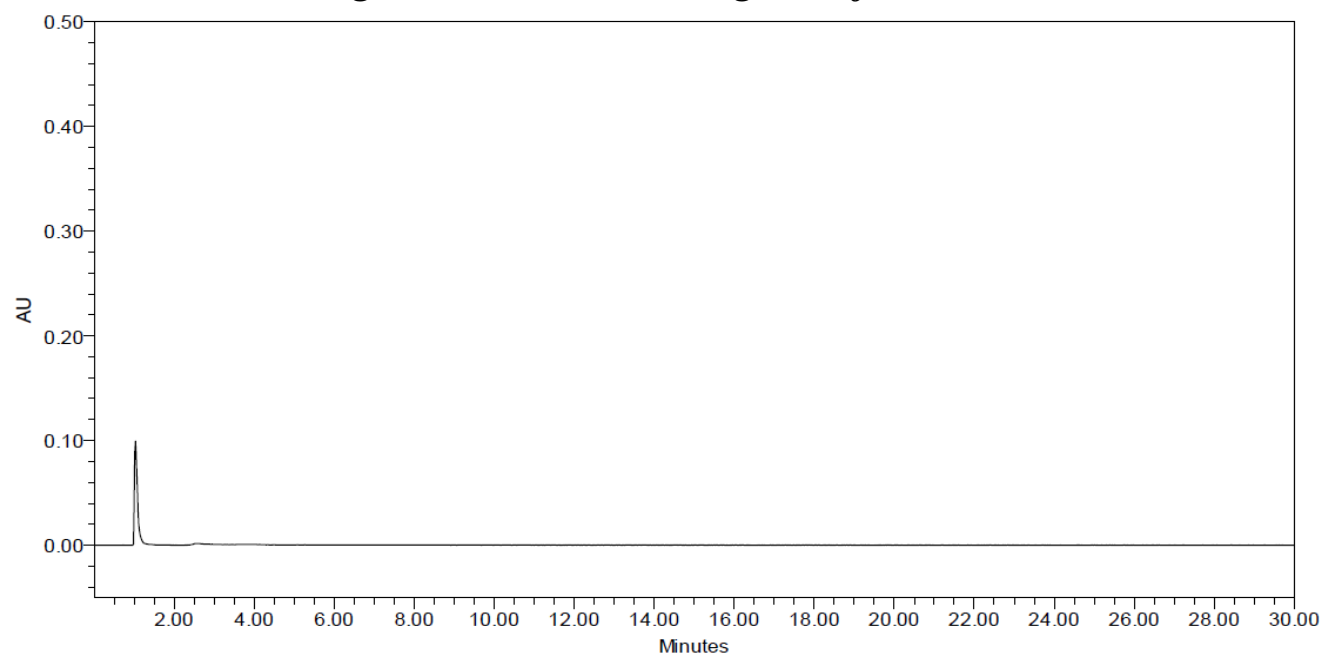

Fig. 5: HPLC Chromatogram of Placebo

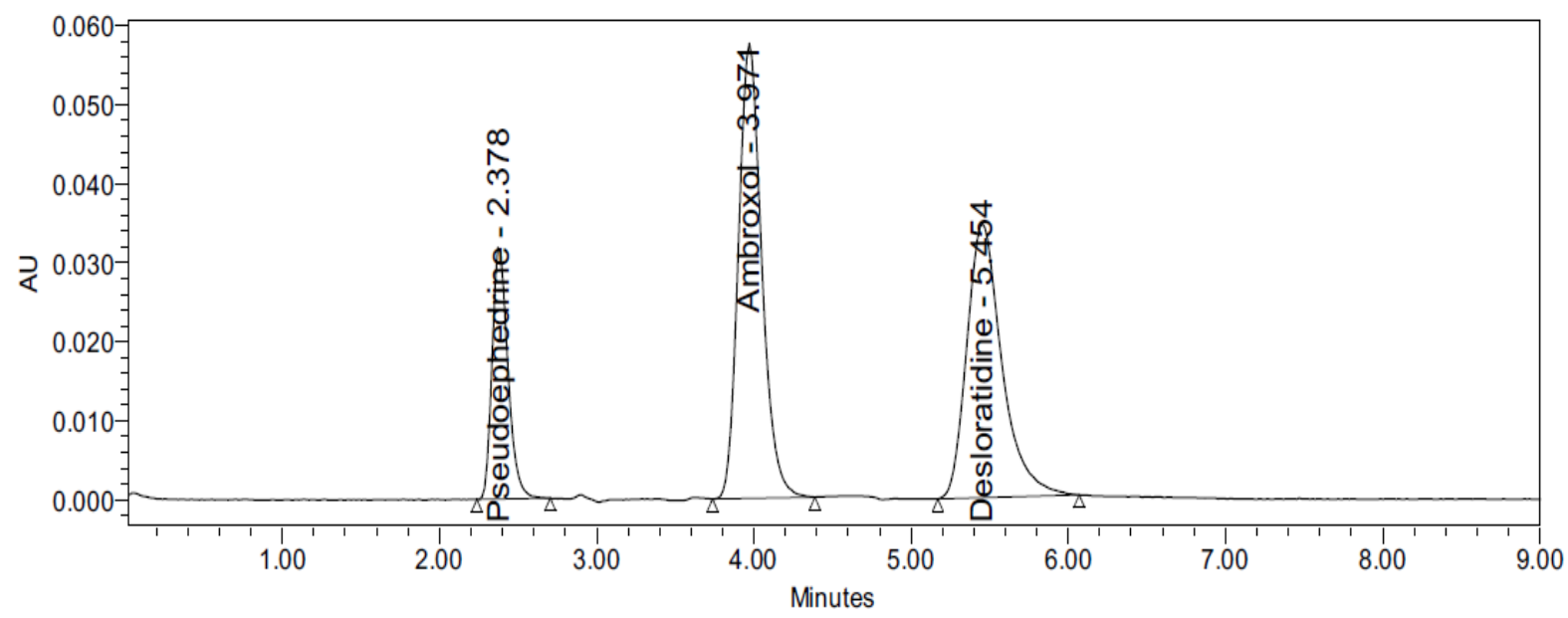

Fig. 6: HPLC Chromatogram of Sample 
Validation of proposed method:

The developed method was validated as per the ICH (International Conference on Harmonization) guidelines with respect to System suitability, Precision, Specificity, Forced degradation studies, Linearity, Accuracy, Limit of detection and Limit of quantification.

\section{Linearity:}

Aliquots of $0.25,0.5,0.75,1.0,1.25$ and $1.5 \mathrm{ml}$ were taken from stock solution of concentration $0.5 \mathrm{mg} / \mathrm{ml}$ Pseudoephedrine, $1.2 \mathrm{mg} / \mathrm{ml}$ Ambroxol, and $0.5 \mathrm{mg} / \mathrm{ml}$ Desloratidine and then diluted up to mark with diluent, such that the final concentrations were in the range $2.5 \mathrm{ppm}-15$ ppm for Pseudoephedrine, 30 ppm-180 ppm for Ambroxol and $2.5 \mathrm{ppm}-15 \mathrm{ppm}$ for Desloratidine. Volume of $10 \mu \mathrm{l}$ of each sample was injected in five times for each concentration level and calibration curve was constructed by plotting the peak area versus drug concentration. A linear relationship between peak area vs. concentration was observed in the range of study. The observations and calibration curve were shown in Table-2 and Fig. 7A-7C.

Table-2: Linearity

\begin{tabular}{ccccccc}
\hline S.No. & $\begin{array}{c}\text { Pseudoephedrine } \\
\text { in ppm }\end{array}$ & $\begin{array}{c}\text { Pseudoephedrine } \\
\text { Area }\end{array}$ & $\begin{array}{c}\text { Conc. of } \\
\text { Ambroxo } \\
\mathbf{1} \text { in ppm }\end{array}$ & $\begin{array}{c}\text { Ambroxol } \\
\text { area }\end{array}$ & $\begin{array}{c}\text { Conc. of } \\
\text { Desloratidine } \\
\text { in ppm }\end{array}$ & $\begin{array}{c}\text { Desloratidine } \\
\text { area }\end{array}$ \\
\hline 1 & 2.5 & 65282 & 30 & 171465 & 2.5 & 134771 \\
2 & 5 & 126557 & 60 & 331741 & 5 & 267448 \\
3 & 7.5 & 179865 & 90 & 487978 & 7.5 & 414170 \\
4 & 10 & 250286 & 120 & 675989 & 10 & 534666 \\
5 & 12.5 & 314103 & 150 & 847462 & 12.5 & 670044 \\
6 & 15 & 367965 & 180 & 988345 & 15 & 809195 \\
\hline
\end{tabular}

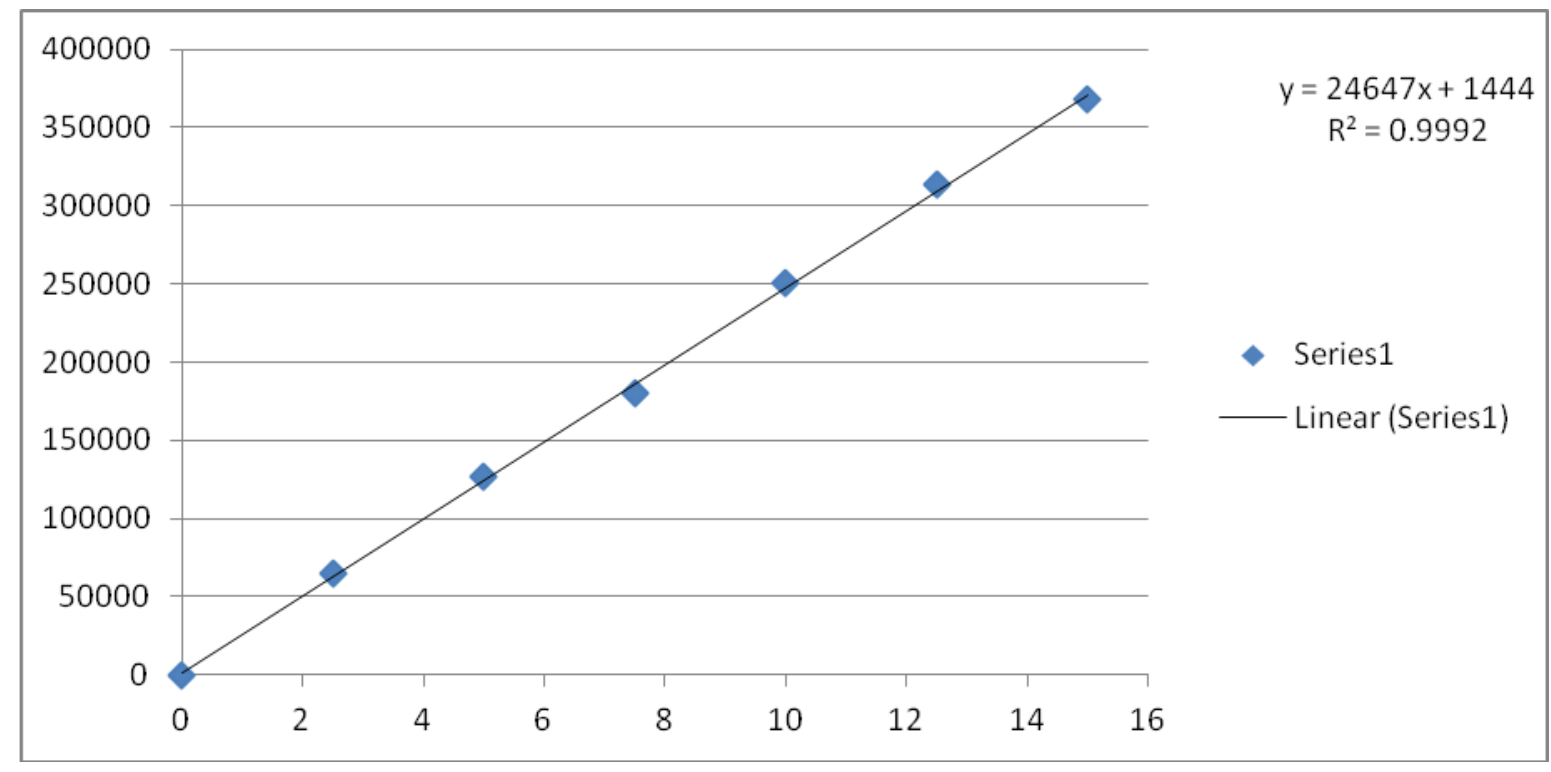

Fig. 7A: Linearity of Pseudoephedrine 


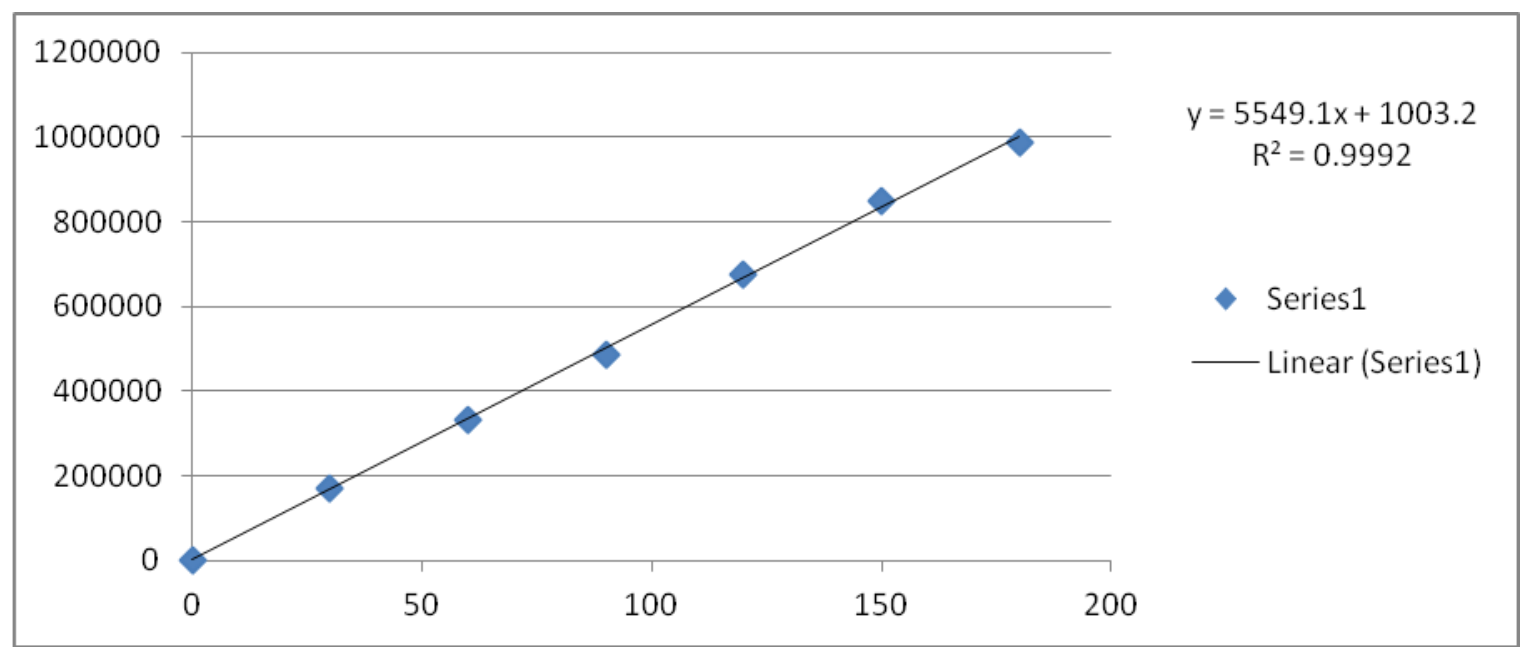

Fig. 7B: Linearity of Ambroxol

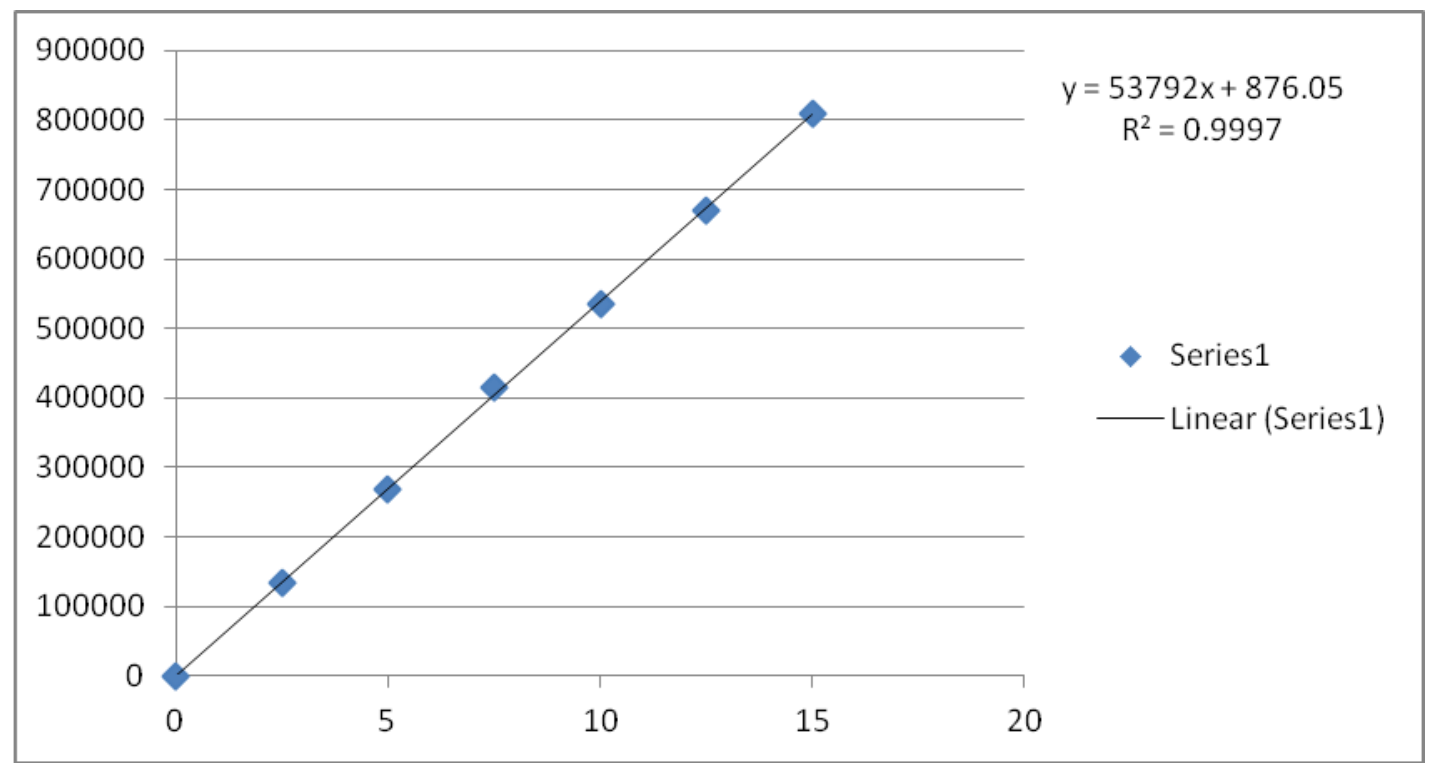

Fig. 7C: Linearity of Desloratidine

\section{System precision:}

Precision is the measure of closeness of the data values to each other for a number of measurements under the same analytical conditions. Standard solution of Pseudo- ephedrine $(10 \mu \mathrm{g} / \mathrm{ml})$, Ambroxol $(120 \mu \mathrm{g} / \mathrm{ml})$ and Desloratidine $(10 \mu \mathrm{g} / \mathrm{ml})$ were prepared as per test method and injected for 6 times. Results are shown in Table-3. 
Table-3: System precision

\begin{tabular}{cccc}
\hline \multirow{2}{*}{ Injections } & \multicolumn{3}{c}{ Areas } \\
\cline { 2 - 4 } & Pseudoephedrine & Ambroxol & Desloratidine \\
\hline $\mathbf{1}$ & 221092 & 597184 & 540160 \\
$\mathbf{2}$ & 220671 & 600606 & 537956 \\
$\mathbf{3}$ & 220894 & 601288 & 537867 \\
$\mathbf{4}$ & 221361 & 598214 & 536842 \\
$\mathbf{5}$ & 222545 & 599337 & 535587 \\
$\mathbf{6}$ & 221357 & 599080 & 530241 \\
AVG & 221320 & 599285 & 536442 \\
SD & 657.0 & 1507.5 & 3390.9 \\
\%RSD & 0.30 & 0.3 & 0.6 \\
\hline
\end{tabular}

Precision:

Intraday and interday precision study of Pseudoephedrine, Ambroxol and Desloratidine were carried out by estimating corresponding responses for 6 times on the same day and on consecutive days for the concentration of $10 \mu \mathrm{g} / \mathrm{ml}$ for Pseudo- ephedrine, $120 \mu \mathrm{g} / \mathrm{ml}$ for Ambroxol and 10 $\mu \mathrm{g} / \mathrm{ml}$ for Desloratidine. The percent relative standard deviation (\%RSD) was calculated which was within the acceptable criteria of not more than 2 . The results were shown in Table-4.

Table-4: Precision

Table-4A: Intra-day precision

\begin{tabular}{cccc}
\hline Sample & \multicolumn{3}{c}{ \%Assay } \\
\cline { 2 - 4 } Preparations & Pseudoephedrine & Ambroxol & Desloratidine \\
\hline Sample-1 & 99.84 & 100.30 & 100.07 \\
Sample-2 & 99.80 & 100.98 & 99.67 \\
Sample-3 & 99.20 & 99.22 & 99.45 \\
Sample-4 & 99.72 & 100.29 & 99.67 \\
Sample-5 & 99.10 & 99.31 & 99.85 \\
Sample-6 & 100.33 & 99.34 & 100.19 \\
\hline AVG & 99.66 & 99.91 & 99.82 \\
SD & 0.454 & 0.72 & 0.275 \\
\%RSD & 0.46 & 0.72 & 0.28 \\
\hline
\end{tabular}

Table-4B: Inter-day precision

\begin{tabular}{cccc}
\hline Sample & \multicolumn{3}{c}{ \%Assay } \\
\cline { 2 - 4 } Preparations & Pseudoephedrine & Ambroxol & Desloratidine \\
\hline Sample-1 & 98.27 & 99.25 & 98.05 \\
Sample-2 & 98.89 & 99.82 & 98.09 \\
Sample-3 & 98.82 & 99.93 & 98.35 \\
Sample-4 & 99.49 & 99.42 & 98.30 \\
Sample-5 & 99.87 & 99.61 & 98.13 \\
Sample-6 & 98.53 & 99.57 & 98.40 \\
AVG & 98.98 & 99.60 & 98.22 \\
S.D & 0.599 & 0.25 & 0.148 \\
\%RSD & 0.60 & 0.25 & 0.15 \\
\hline
\end{tabular}




\section{Accuracy (Recovery studies):}

To determine the accuracy in sample preparation, method of standard additions was made for measuring the recovery of the drugs. A fixed amount of sample was taken and standard drug was added at 50\%, 100\% and $150 \%$ levels. The results were analyzed and the results were found to be within the limits. The accuracy was expressed as the percentage of the analyte recovery. The results were shown in Table-5.

\section{Table-5: Accuracy}

\begin{tabular}{|c|c|c|c|c|c|c|c|c|c|}
\hline \multirow[b]{2}{*}{ \% Conc. } & \multicolumn{3}{|c|}{ Pseudoephedrine } & \multicolumn{3}{|c|}{ Ambroxol } & \multicolumn{3}{|c|}{ Desloratidine } \\
\hline & $\begin{array}{l}\text { Amount } \\
\text { Added } \\
(\mu \mathrm{g} / \mathrm{ml})\end{array}$ & $\begin{array}{l}\text { Amount } \\
\text { Found } \\
(\mu \mathrm{g} / \mathrm{ml})\end{array}$ & \% Recovery & $\begin{array}{l}\text { Amount } \\
\text { Added } \\
(\boldsymbol{\mu g} / \mathbf{m l})\end{array}$ & $\begin{array}{l}\text { Amount } \\
\text { Found } \\
(\boldsymbol{\mu g} / \mathbf{m l})\end{array}$ & \%Recovery & $\begin{array}{c}\text { Amount } \\
\text { Added } \\
(\mu \mathrm{g} / \mathrm{ml})\end{array}$ & $\begin{array}{l}\text { Amount } \\
\text { Found } \\
(\boldsymbol{\mu g} / \mathbf{m l})\end{array}$ & \% Recovery \\
\hline 50 & 5 & 4.98 & 99.68 & 60 & 59.75 & 99.58 & 5 & 4.97 & 99.41 \\
\hline 50 & 5 & 5.00 & 99.94 & 60 & 59.66 & 99.43 & 5 & 5.01 & 100.10 \\
\hline 50 & 5 & 5.08 & 101.62 & 60 & 59.48 & 99.13 & 5 & 5.05 & 100.98 \\
\hline 100 & 10 & 10.19 & 101.89 & 120 & 120.73 & 100.61 & 10 & 10.08 & 100.81 \\
\hline 100 & 10 & 9.91 & 99.10 & 120 & 119.33 & 99.44 & 10 & 10.06 & 100.60 \\
\hline 100 & 10 & 9.97 & 99.72 & 120 & 119.75 & 99.79 & 10 & 9.90 & 99.00 \\
\hline 150 & 15 & 14.92 & 99.47 & 180 & 180.69 & 100.38 & 15 & 15.12 & 100.81 \\
\hline 150 & 15 & 14.93 & 99.50 & 180 & 179.38 & 99.65 & 15 & 14.94 & 99.62 \\
\hline \multirow[t]{4}{*}{150} & 15 & 14.94 & 99.63 & 180 & 182.02 & 101.12 & 15 & 15.19 & 101.27 \\
\hline & \multicolumn{2}{|c|}{ Average } & 100.06 & \multicolumn{2}{|c|}{ Average } & 99.90 & \multicolumn{2}{|c|}{ Average } & 100.29 \\
\hline & \multicolumn{2}{|c|}{$\mathrm{SD}$} & 0.99 & \multicolumn{2}{|c|}{ SD } & 0.65 & \multicolumn{2}{|c|}{$\mathrm{SD}$} & 0.79 \\
\hline & \multicolumn{2}{|c|}{ RSD } & 0.99 & \multicolumn{2}{|c|}{ RSD } & 0.65 & \multicolumn{2}{|c|}{ RSD } & 0.79 \\
\hline
\end{tabular}

$\mathrm{SD}=$ Standard Deviation; RSD = Relative Standard Deviation.

\section{Specificity:}

The specificity of the method was performed by injecting blank solution (without any sample) and then a drug solution of $10 \mu \mathrm{l}$ injected into the column, under optimized chromatographic conditions, to demonstrate the separation of three molecules Pseudoephedrine, Ambroxol and Desloratidine from any of the impurities, if present. As there was no interference of impurities, the method was found to be specific.
Limit of Detection and Limit of Quantification:

LOD and LOQ were calculated using the following formula $\mathrm{LOD}=3.3(\mathrm{SD}) / \mathrm{S}$ and $\mathrm{LOQ}=$ $10(\mathrm{SD}) / \mathrm{S}$, where SD = standard deviation of response (peak area) and $S=$ slope of the calibration curve. Limit of Detection and Limit of Quantification were found to be $0.041 \mu \mathrm{g} / \mathrm{ml}$ and $0.125 \mu \mathrm{g} / \mathrm{ml}$ respectively for Pseudoephedrine, $0.143 \mu \mathrm{g} / \mathrm{ml}$ and 0.435 $\mu \mathrm{g} / \mathrm{ml}$ respectively for Ambroxol and 0.034 $\mu \mathrm{g} / \mathrm{ml}$ and $0.103 \mu \mathrm{g} / \mathrm{ml}$ respectively for Desloratidine as per ICH guidelines. The results were shown in Table- 6 and chromatograms were shown in Fig. 8A \& 8B. 
Table-6: Characteristics of HPLC method

\begin{tabular}{|c|c|c|}
\hline Drug & Parameters defined & Obtained value \\
\hline \multirow{8}{*}{ Pseudoephedrine } & Linearity range (ppm) & $2.5-15 \mathrm{ppm}$ \\
\hline & Regression coefficient $\left(\mathrm{r}^{2}\right)$ & 0.999 \\
\hline & Intercept & 1444 \\
\hline & Slope & 24647 \\
\hline & LOD (ppm) & 0.041 \\
\hline & LOQ (ppm) & 0.125 \\
\hline & Tailing factor & 1.37 \\
\hline & Plate count & 3120 \\
\hline \multirow{8}{*}{ Ambroxol } & Linearity range (ppm) & 30-180 ppm \\
\hline & Regression coefficient $\left(\mathrm{r}^{2}\right)$ & 0.999 \\
\hline & Intercept & 1003 \\
\hline & Slope & 5549 \\
\hline & LOD (ppm) & 0.143 \\
\hline & LOQ (ppm) & 0.435 \\
\hline & Tailing factor & 1.23 \\
\hline & Plate count & 4016 \\
\hline \multirow{8}{*}{ Desloratidine } & Linearity range $(\mathrm{ppm})$ & $2.5-15 \mathrm{ppm}$ \\
\hline & Regression coefficient $\left(\mathrm{r}^{2}\right)$ & 0.999 \\
\hline & Intercept & 876 \\
\hline & Slope & 53792 \\
\hline & LOD (ppm) & 0.034 \\
\hline & LOQ (ppm) & 0.103 \\
\hline & Tailing factor & 1.45 \\
\hline & Plate count & 3651 \\
\hline
\end{tabular}

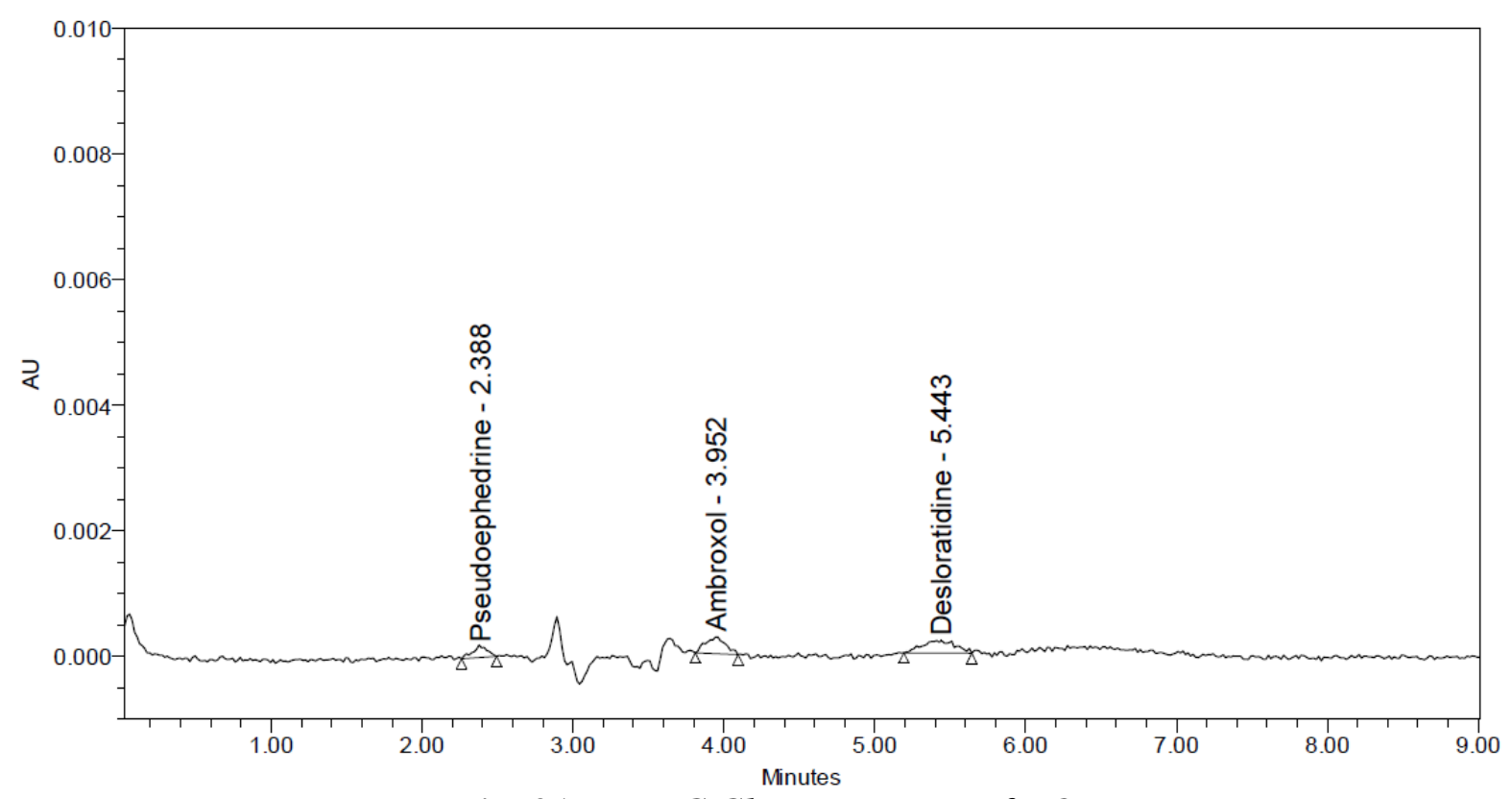

Fig. 8A: HPLC Chromatogram of LOD 


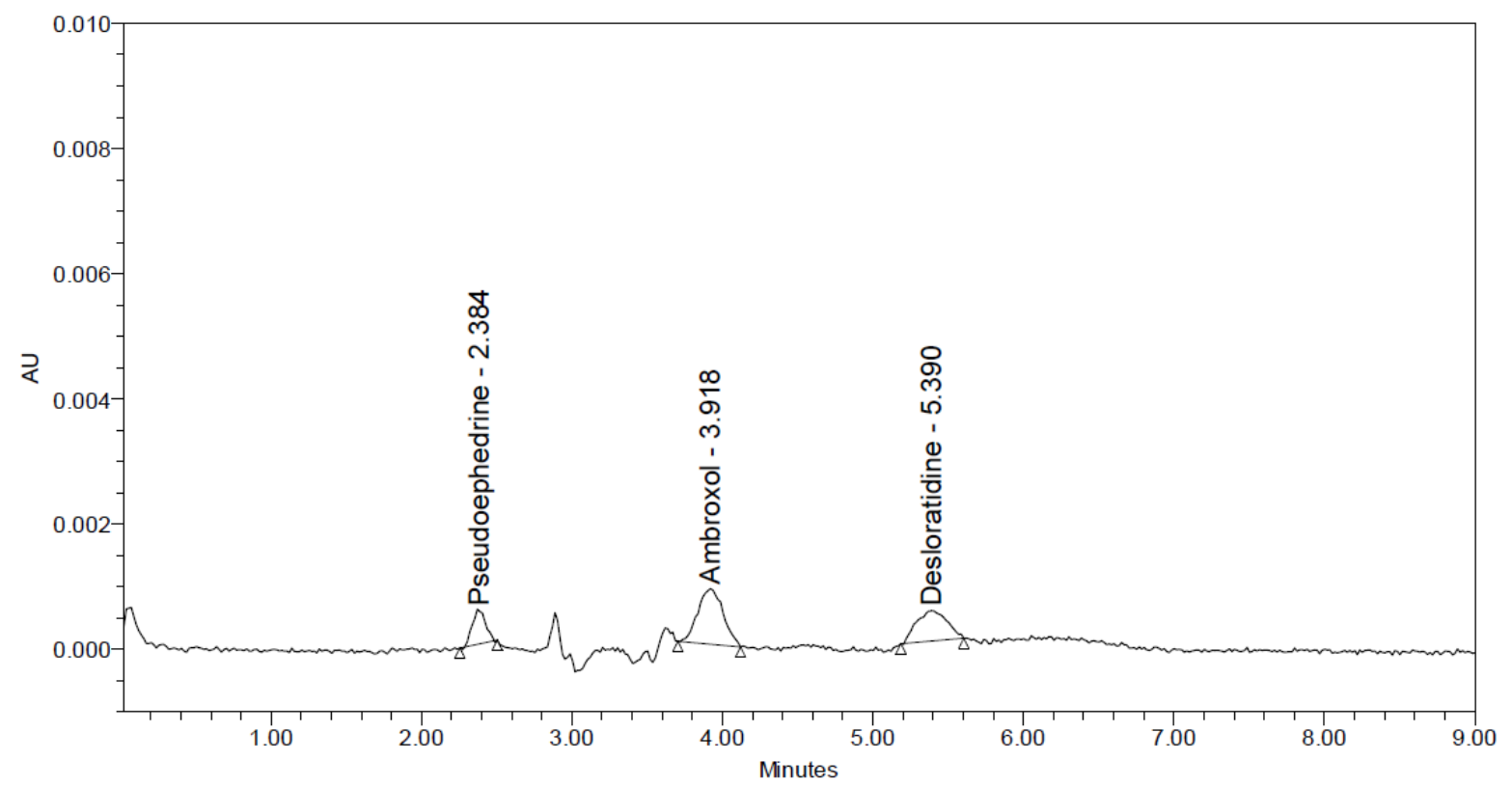

Fig. 8B: HPLC Chromatogram of LOQ

Robustness:

Robustness was carried by varying three parameters from the optimized chromatographic conditions such as making small changes in flow rate $( \pm 0.1 \mathrm{ml} / \mathrm{min})$, mobile phase composition $( \pm 5 \%)$ and column temperature $\left( \pm 5^{\circ} \mathrm{C}\right)$. It was observed that the small changes in these operational parameters did not lead to changes of retention time of the peak of interest and the \%RSD was not more than 2.0. The degree of reproducibility of the results proven that the method is robust. The results were shown in Table-7A-7C.

Table-7A: Robustness of Pseudoephedrine

\begin{tabular}{|c|c|c|c|c|c|c|c|}
\hline S.No. & Parameter & Optimized & Used & $\begin{array}{l}\text { Peak } \\
\text { Area }\end{array}$ & $\begin{array}{c}\text { Retention } \\
\text { Time }\end{array}$ & $\begin{array}{l}\text { Plate } \\
\text { count }\end{array}$ & $\begin{array}{l}\text { Tailing } \\
\text { Factor }\end{array}$ \\
\hline \multirow{3}{*}{1.} & \multirow{3}{*}{$\begin{array}{l}\text { Flow Rate } \\
( \pm 0.1 \mathrm{ml} / \mathrm{min})\end{array}$} & \multirow{3}{*}{$1.0 \mathrm{ml} / \mathrm{min}$} & $0.9 \mathrm{ml} / \mathrm{min}$ & 245494 & 2.665 & 2578 & 1.30 \\
\hline & & & $1.0 \mathrm{ml} / \mathrm{min}$ & 221092 & 2.375 & 3120 & 1.37 \\
\hline & & & $1.1 \mathrm{ml} / \mathrm{min}$ & 200865 & 2.146 & 2044 & 1.24 \\
\hline \multirow{3}{*}{2.} & \multirow{3}{*}{$\begin{array}{l}\text { Column } \\
\text { Temperature } \\
\quad\left( \pm 5^{\circ} \mathrm{C}\right)\end{array}$} & \multirow{3}{*}{$30^{\circ} \mathrm{C}$} & $25^{\circ} \mathrm{C}$ & 243382 & 2.665 & 2589 & 1.29 \\
\hline & & & $30^{\circ} \mathrm{C}$ & 221092 & 2.375 & 3120 & 1.37 \\
\hline & & & $35^{\circ} \mathrm{C}$ & 198528 & 2.146 & 2055 & 1.22 \\
\hline \multirow{3}{*}{3.} & \multirow{3}{*}{$\begin{array}{l}\text { Mobile phase } \\
\text { composition }\end{array}$} & \multirow{3}{*}{$\begin{array}{c}\text { Buffer : ACN : Methanol } \\
\text { 50:20:30 v/v }\end{array}$} & $55: 20: 25$ & 246518 & 2.377 & 2365 & 1.27 \\
\hline & & & $50: 20: 30$ & 221092 & 2.375 & 3120 & 1.37 \\
\hline & & & $45: 20: 35$ & 230506 & 2.375 & 2386 & 1.27 \\
\hline
\end{tabular}


Table-7B: Robustness of Ambroxol

\begin{tabular}{|c|c|c|c|c|c|c|c|}
\hline S.No. & Parameter & Optimized & Used & $\begin{array}{l}\text { Peak } \\
\text { Area }\end{array}$ & $\begin{array}{c}\text { Retention } \\
\text { Time }\end{array}$ & $\begin{array}{l}\text { Plate } \\
\text { count }\end{array}$ & $\begin{array}{l}\text { Tailing } \\
\text { Factor }\end{array}$ \\
\hline \multirow{3}{*}{1.} & \multirow{3}{*}{$\begin{array}{l}\text { Flow Rate } \\
( \pm 0.1 \mathrm{ml} / \mathrm{min})\end{array}$} & \multirow{3}{*}{$1.0 \mathrm{ml} / \mathrm{min}$} & $0.9 \mathrm{ml} / \mathrm{min}$ & 609374 & 4.391 & 3050 & 1.17 \\
\hline & & & $1.0 \mathrm{ml} / \mathrm{min}$ & 597184 & 3.971 & 4016 & 1.23 \\
\hline & & & $1.1 \mathrm{ml} / \mathrm{min}$ & 506876 & 3.534 & 2457 & 1.14 \\
\hline \multirow{3}{*}{2.} & \multirow{3}{*}{$\begin{array}{l}\text { Column } \\
\text { Temperature } \\
\left( \pm 5^{\circ} \mathrm{C}\right)\end{array}$} & \multirow{3}{*}{$30^{\circ} \mathrm{C}$} & $25^{\circ} \mathrm{C}$ & 608241 & 4.391 & 3053 & 1.17 \\
\hline & & & $30^{\circ} \mathrm{C}$ & 597184 & 3.971 & 4016 & 1.23 \\
\hline & & & $35^{\circ} \mathrm{C}$ & 500539 & 3.534 & 2467 & 1.15 \\
\hline \multirow{3}{*}{3.} & \multirow{3}{*}{$\begin{array}{l}\text { Mobile phase } \\
\text { composition }\end{array}$} & \multirow{3}{*}{$\begin{array}{c}\text { Buffer : ACN : Methanol } \\
\text { 50:20:30 v/v }\end{array}$} & $55: 20: 25$ & 668908 & 3.911 & 2785 & 1.14 \\
\hline & & & $50: 20: 30$ & 597184 & 3.971 & 4016 & 1.23 \\
\hline & & & $45: 20: 35$ & 596848 & 3.918 & 2780 & 1.14 \\
\hline
\end{tabular}

Table-7C: Robustness of Desloratidine

\begin{tabular}{|c|c|c|c|c|c|c|c|}
\hline S.No. & Parameter & Optimized & Used & $\begin{array}{l}\text { Peak } \\
\text { Area } \\
\end{array}$ & $\begin{array}{c}\text { Retention } \\
\text { Time }\end{array}$ & $\begin{array}{l}\text { Plate } \\
\text { count }\end{array}$ & $\begin{array}{l}\text { Tailing } \\
\text { Factor } \\
\end{array}$ \\
\hline \multirow{3}{*}{1.} & \multirow{3}{*}{$\begin{array}{l}\text { Flow Rate } \\
\qquad( \pm 0.1 \\
\mathrm{ml} / \mathrm{min})\end{array}$} & \multirow{3}{*}{$1.0 \mathrm{ml} / \mathrm{min}$} & $0.9 \mathrm{ml} / \mathrm{min}$ & 587083 & 6.029 & 2719 & 1.32 \\
\hline & & & $1.0 \mathrm{ml} / \mathrm{min}$ & 540160 & 5.448 & 3651 & 1.45 \\
\hline & & & $1.1 \mathrm{ml} / \mathrm{min}$ & 485894 & 4.863 & 2161 & 1.29 \\
\hline \multirow{3}{*}{2.} & \multirow{3}{*}{$\begin{array}{c}\text { Column } \\
\text { Temperature } \\
\left( \pm 5^{\circ} \mathrm{C}\right)\end{array}$} & \multirow{3}{*}{$30^{\circ} \mathrm{C}$} & $25^{\circ} \mathrm{C}$ & 578794 & 6.029 & 2737 & 1.30 \\
\hline & & & $30^{\circ} \mathrm{C}$ & 540160 & 5.448 & 3651 & 1.45 \\
\hline & & & $35^{\circ} \mathrm{C}$ & 470597 & 4.863 & 2192 & 1.22 \\
\hline \multirow{3}{*}{3.} & \multirow{3}{*}{$\begin{array}{c}\text { Mobile } \\
\text { phase } \\
\text { composition }\end{array}$} & \multirow{3}{*}{$\begin{array}{c}\text { Buffer : ACN : } \\
\text { Methanol } \\
50: 20: 30 \mathrm{v} / \mathrm{v}\end{array}$} & $55: 20: 25$ & 604648 & 5.372 & 2510 & 1.32 \\
\hline & & & $50: 20: 30$ & 540160 & 5.448 & 3651 & 1.45 \\
\hline & & & $45: 20: 35$ & 556599 & 5.382 & 2491 & 1.31 \\
\hline
\end{tabular}

\section{System suitability \& System precision:}

The system suitability was determined by making six replicate injections from freshly prepared standard solutions. The observed RSD values were well within usually accepted limits $(\leq 2 \%)$. Theoretical plates, tailing factor of Pseudoephedrine, Ambroxol and Desloratidinewere determined. The results are all within acceptable limits summarized in Table-3 \& 6.

\section{Forced Degradation Studies:}

Forced degradation studies were performed to demonstrate the optimized method is stability indicating. And to prove the method which can be able to measure accurately active pharmaceutical ingredient in presence of degradants which are expected to be formed during different types of degradations www.ijapbjournal.com applied to the drug sample. Forced degradation conditions were optimized during method development. Final optimized forced degradation conditions are as follows For forced degradation analysis, aliquots of stock $(0.1 \mathrm{mg} / \mathrm{ml}, 1.2 \mathrm{mg} / \mathrm{ml}$ and $0.1 \mathrm{mg} / \mathrm{ml})$ were prepared separately and treated with 1 $\mathrm{ml}$ of $2 \mathrm{~N} \mathrm{HCl}$ (Acid stability), $1 \mathrm{ml}$ of $2 \mathrm{~N} \mathrm{NaOH}$ (Alkaline stability), $1 \mathrm{ml}$ of $30 \% \quad \mathrm{H}_{2} \mathrm{O}_{2}$ (Oxidative degradation), exposure of standard drug solution at $105^{\circ} \mathrm{C}$ for $5 \mathrm{~h}$ (dry heat degradation), photo stability degradation (exposure of drug at 200 watt $/ \mathrm{m}^{2}$ ) and neutral degradation (refluxing with water at $60^{\circ} \mathrm{C}$ for $8 \mathrm{~h}$. Stability of these samples was compared with fresh sample on the day of analysis. The HPLC chromatograms of degraded products show no interference at the analyte peaks, hence the method was 
specific and stability indicating. The results were shown in Table-8 and the chromatograms were shown in Fig. 9A-9F.
The detailed degradation for each condition is as follows:

\section{Table-8: Forced Degradation Studies}

Table-8A: Forced Degradation Studies for Pseudoephedrine

\begin{tabular}{ccccccc}
\hline S.No & Injection & \%Assay & \%Degradation & $\begin{array}{c}\text { Purity } \\
\text { Angle }\end{array}$ & $\begin{array}{c}\text { Purity } \\
\text { Threshold }\end{array}$ & $\begin{array}{c}\text { Purity } \\
\text { Flag }\end{array}$ \\
\hline 1. & $\begin{array}{c}\text { Controlled } \\
\text { sample }\end{array}$ & 99.66 & -- & 0.987 & 1.456 & No \\
Acid & $\begin{array}{c}\text { Acid } \\
\text { Degradation }\end{array}$ & 94.59 & 5.09 & 0.834 & 1.170 & No \\
Base & 95.81 & 3.86 & 0.135 & 0.215 & No \\
4. & $\begin{array}{c}\text { Degradation } \\
\text { Peroxide }\end{array}$ & 92.61 & 7.07 & 1.565 & 5.926 & No \\
5. & $\begin{array}{c}\text { Degradation } \\
\text { Thermal }\end{array}$ & 97.45 & 2.22 & 1.253 & 4.345 & No \\
6. & $\begin{array}{c}\text { Degradation } \\
\text { UV Degradation } \\
\text { 7. }\end{array}$ & 98.55 & 1.11 & 1.134 & 3.546 & No \\
\hline
\end{tabular}

Table-8B: Forced Degradation Studies for Ambroxol

\begin{tabular}{ccccccc}
\hline S.No. & Injection & \%Assay & \%Degradation & $\begin{array}{c}\text { Purity } \\
\text { Angle }\end{array}$ & $\begin{array}{c}\text { Purity } \\
\text { Threshold }\end{array}$ & $\begin{array}{c}\text { Purity } \\
\text { Flag }\end{array}$ \\
\hline 1. & $\begin{array}{c}\text { Controlled } \\
\text { sample } \\
\text { Acid }\end{array}$ & 99.91 & -- & 0.556 & 1.211 & No \\
2. & 93.64 & 6.28 & 1.102 & 1.402 & No \\
3egradation & $\begin{array}{c}\text { Base } \\
\text { Degradation }\end{array}$ & 96.01 & 3.90 & 0.154 & 0.256 & No \\
4. & $\begin{array}{c}\text { Peroxide } \\
\text { Degradation } \\
\text { Thermal }\end{array}$ & 94.03 & 5.89 & 1.093 & 2.854 & No \\
5. & 96.89 & 3.02 & 1.345 & 2.835 & No \\
6. & $\begin{array}{c}\text { Degradation } \\
\text { UV Degradation } \\
\text { W. }\end{array}$ & 98.77 & 1.14 & 1.123 & 2.456 & No \\
\hline
\end{tabular}


Table-8C: Forced Degradation Studies for Desloratidine

\begin{tabular}{ccccccc}
\hline S.No. & Injection & \%Assay & \%Degradation & $\begin{array}{c}\text { Purity } \\
\text { Angle }\end{array}$ & $\begin{array}{c}\text { Purity } \\
\text { Threshold }\end{array}$ & $\begin{array}{c}\text { Purity } \\
\text { Flag }\end{array}$ \\
\hline 1. & $\begin{array}{c}\text { Controlled } \\
\text { Sample } \\
\text { Acid }\end{array}$ & 99.82 & -- & 0.867 & 1.231 & No \\
2. & 93.47 & 6.36 & 0.192 & 0.323 & No \\
Degradation & Base & 93.22 & 6.61 & 0.185 & 0.308 & No \\
4. & $\begin{array}{c}\text { Degradation } \\
\text { Peroxide }\end{array}$ & 92.59 & 7.24 & 0.190 & 0.334 & No \\
5. & $\begin{array}{c}\text { Degradation } \\
\text { Thermal }\end{array}$ & 95.37 & 4.46 & 0.343 & 1.324 & No \\
6. & $\begin{array}{c}\text { Degradation } \\
\text { UV Degradation } \\
\text { W. }\end{array}$ & 97.36 & 2.46 & 0.176 & 0.338 & No \\
\hline
\end{tabular}

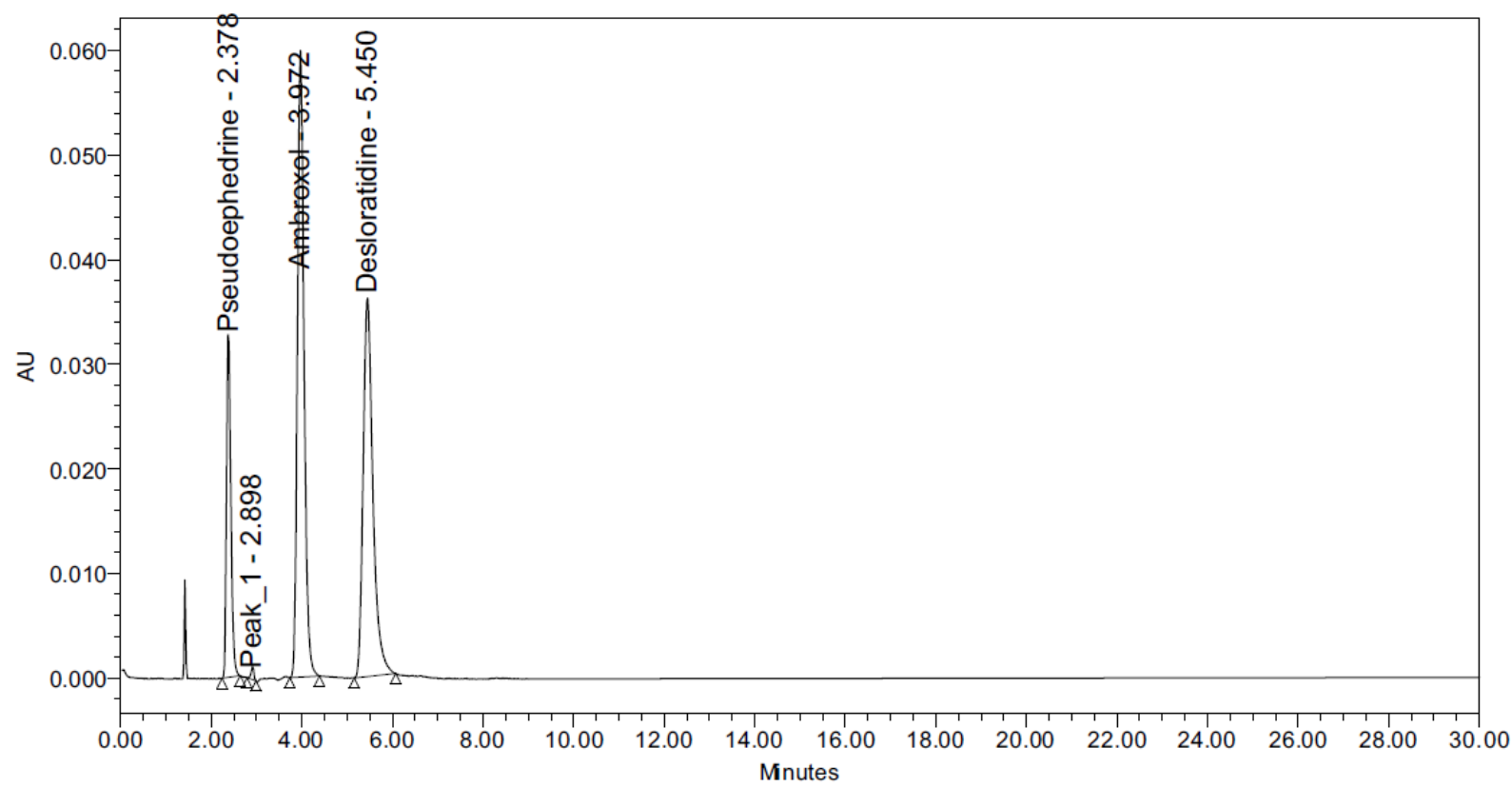

Fig. 9A: Acid Degradation 


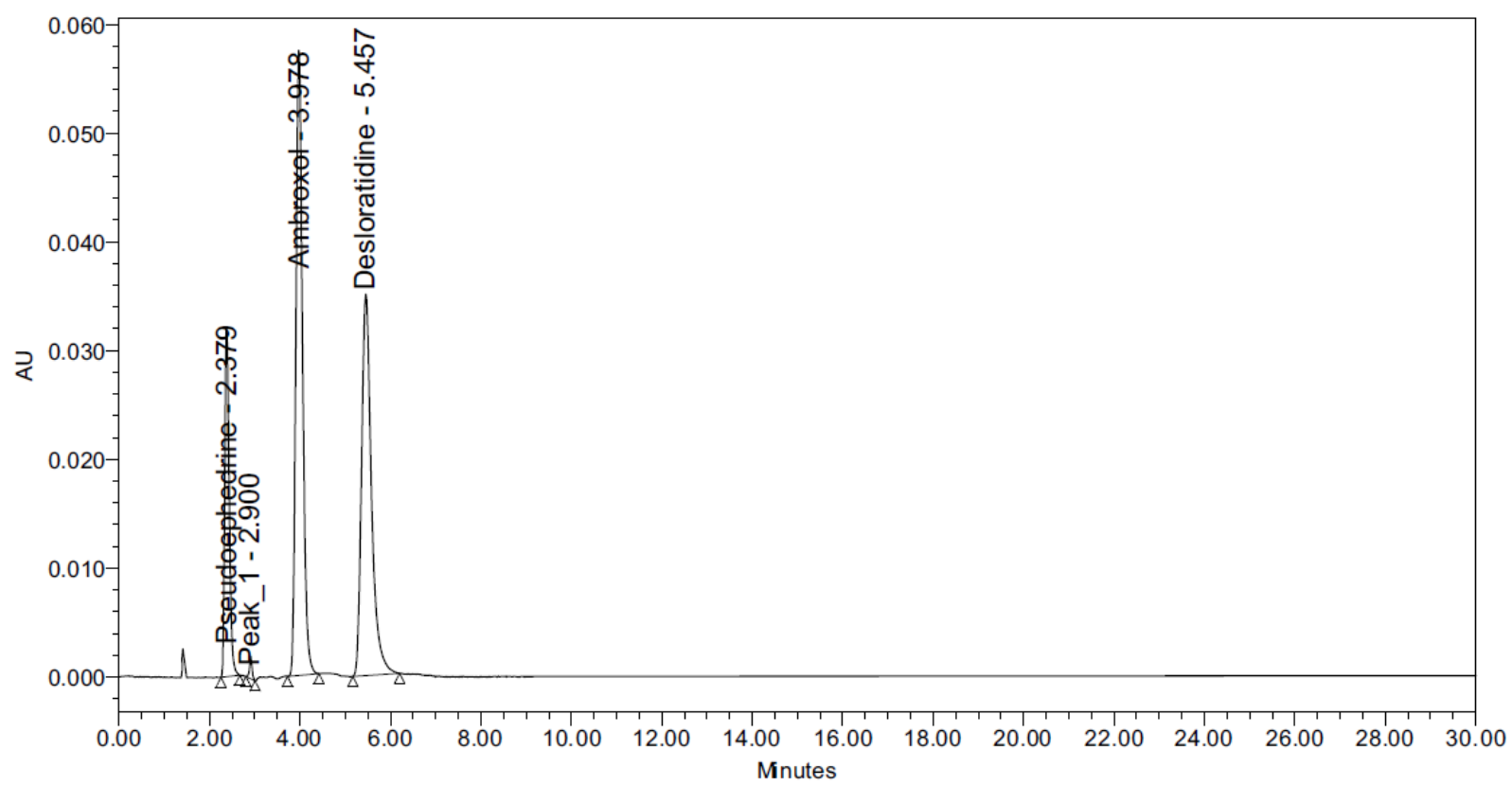

Fig. 9B: Base Degradation

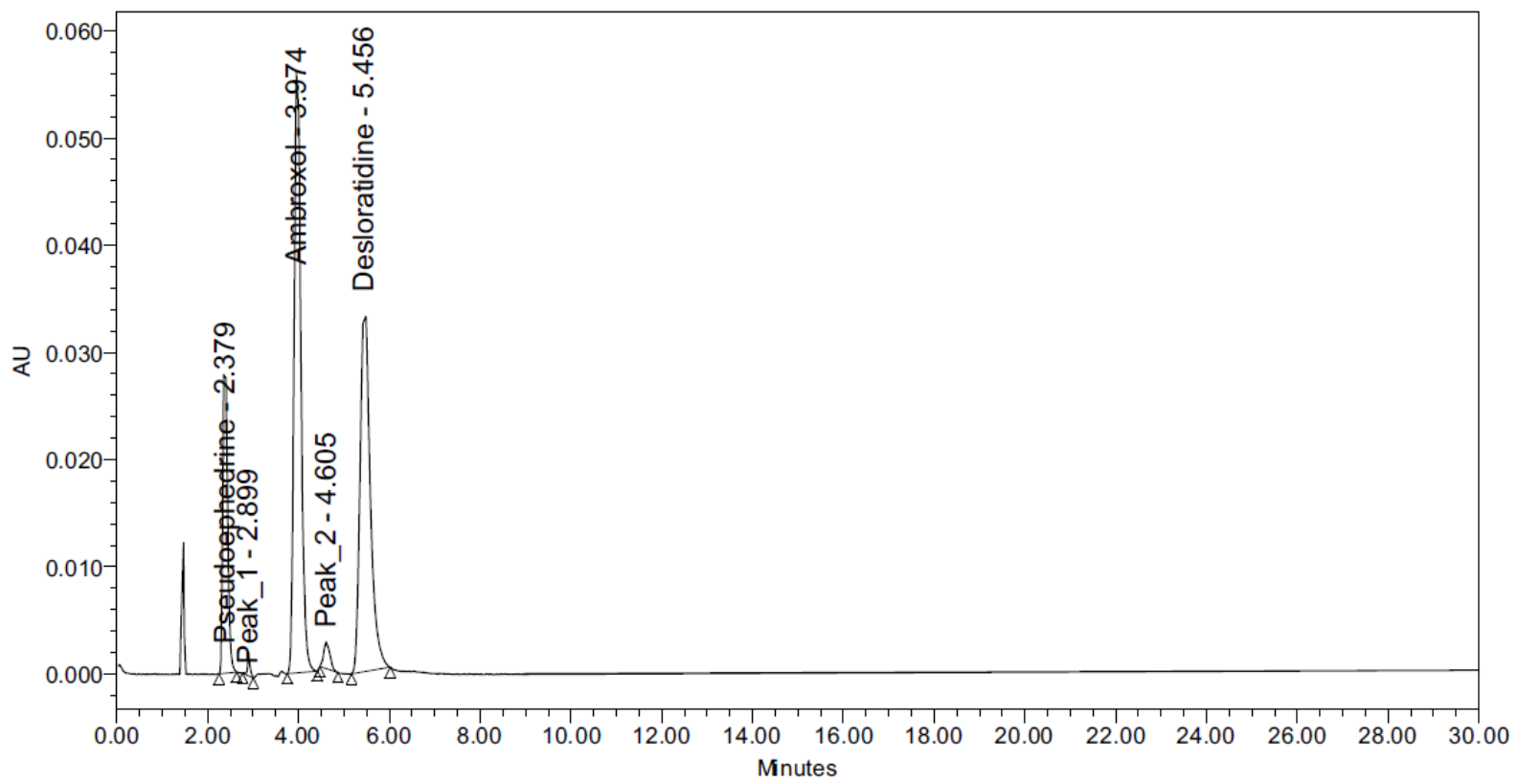

Fig. 9C: Peroxide Degradation 


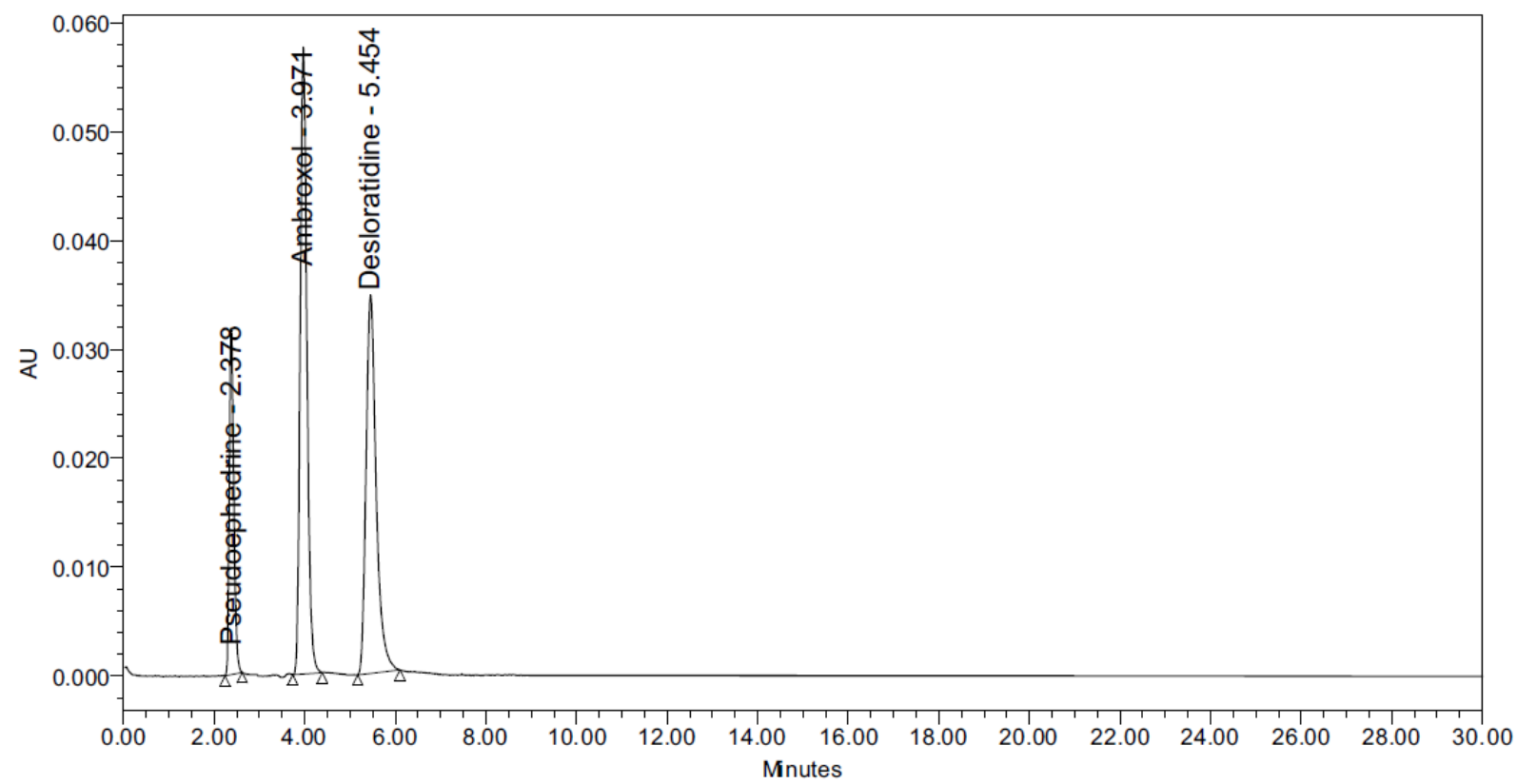

Fig. 9D: Thermal Degradation

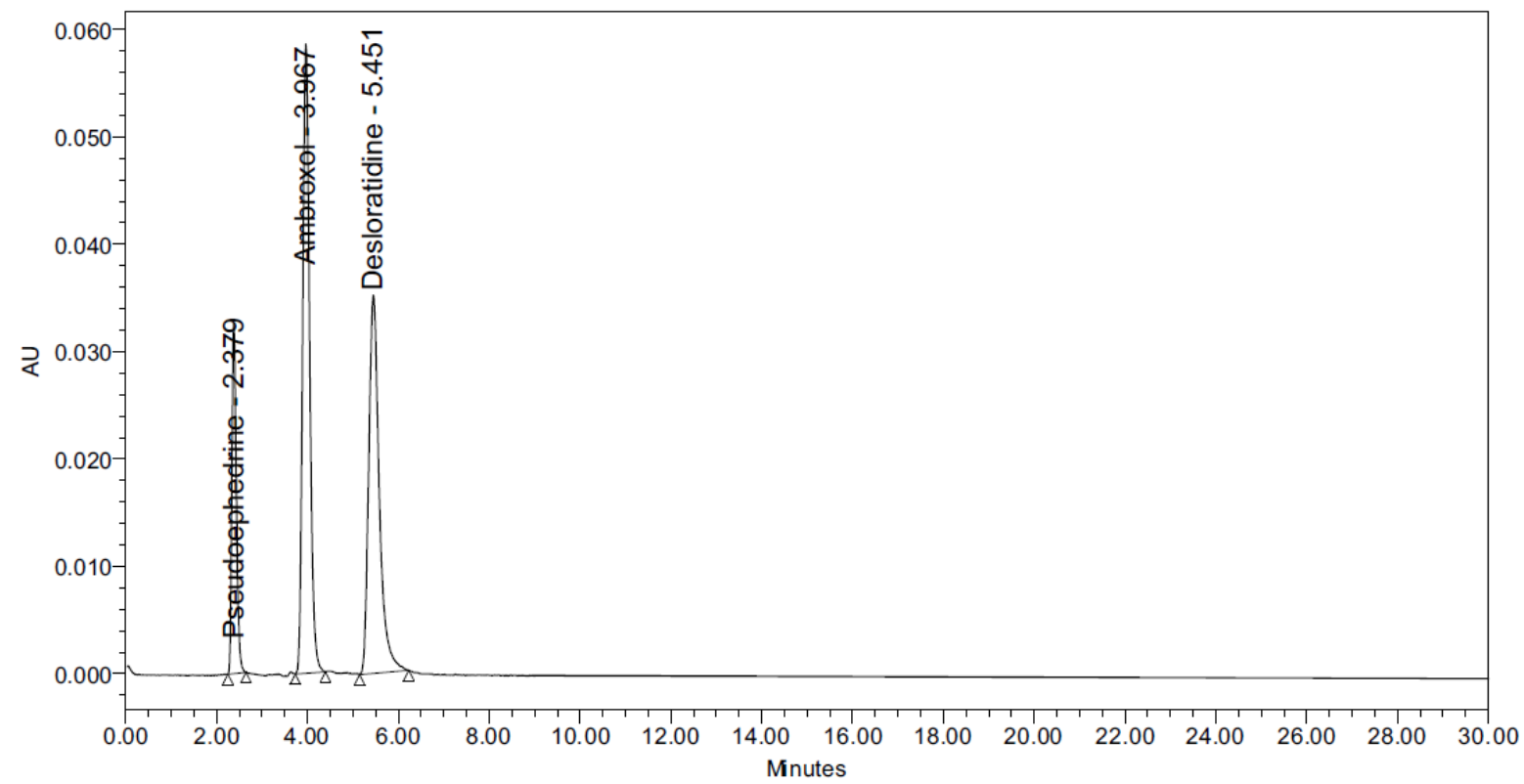

Fig. 9E: UV Degradation 


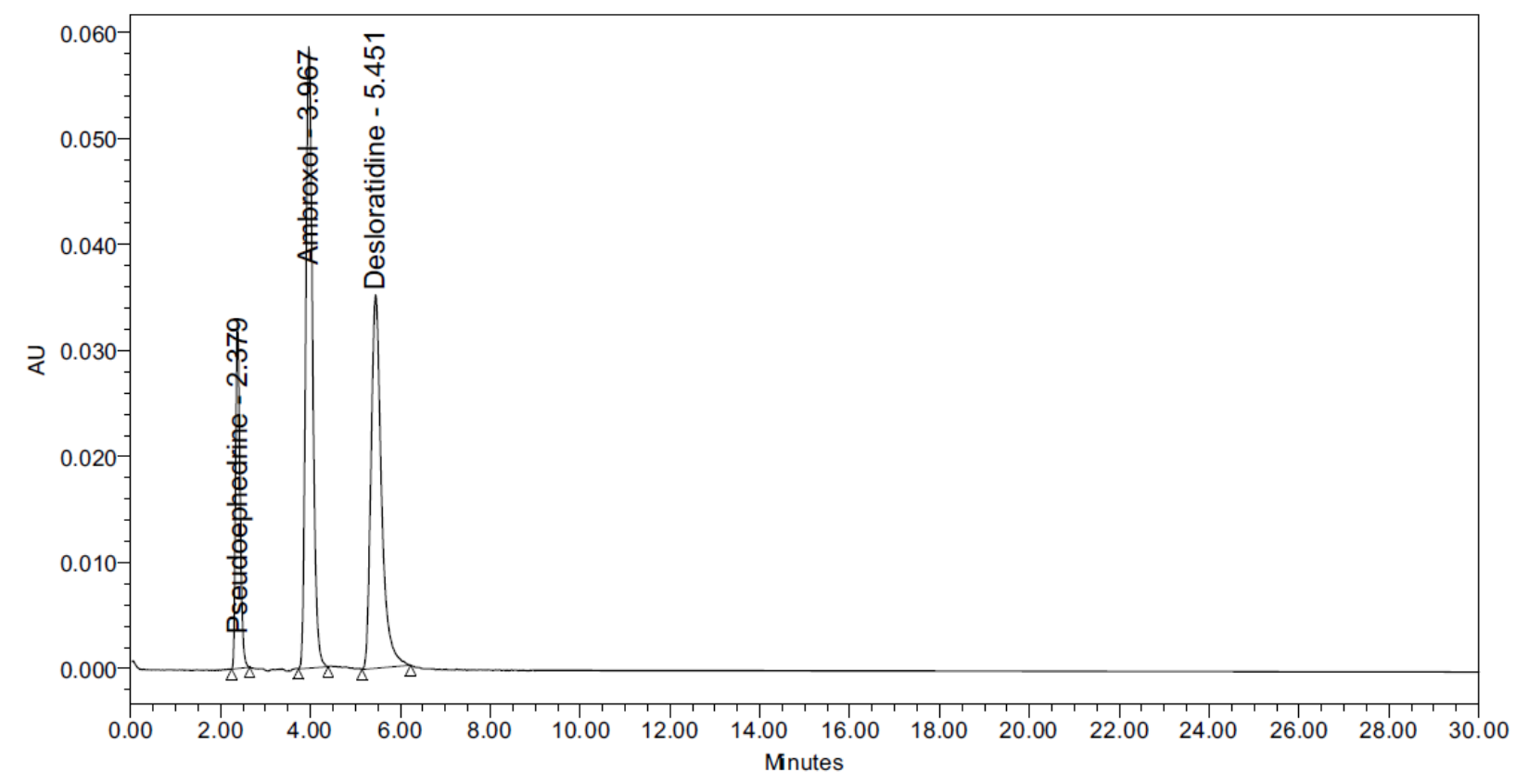

Fig. 9F: Water degradation

\section{Oxidation:}

To $1 \mathrm{ml}$ of stock solution of Pseudoephedrine, Ambroxol and Desloratidine, $1 \mathrm{ml}$ of $30 \%$ hydrogen peroxide $\left(\mathrm{H}_{2} \mathrm{O}_{2}\right)$ was added separately. The solutions were kept for $30 \mathrm{~min}$ at $60^{\circ} \mathrm{C}$. For HPLC study, the resultant solution was diluted to obtain $60 \mu \mathrm{g} / \mathrm{ml}, 120 \mu \mathrm{g} / \mathrm{ml}$ and $10 \mu \mathrm{g} / \mathrm{ml}$ solution for Pseudoephedrine, Ambroxol and Desloratidine respectively and $10 \mu \mathrm{l}$ were injected into the system and the chromatograms were recorded to assess the stability of sample.

\section{Acid Degradation Studies:}

To $1 \mathrm{ml}$ of stock solution Pseudoephedrine, Ambroxol and Desloratidine, $1 \mathrm{ml}$ of $2 \mathrm{~N}$ Hydrochloric acid was added and refluxed for $30 \mathrm{mins}$ at $60^{\circ} \mathrm{C}$. The resultant solution was diluted to obtain $60 \mu \mathrm{g} / \mathrm{ml}, 120 \mu \mathrm{g} / \mathrm{ml}$ and $10 \mu \mathrm{g} / \mathrm{ml}$ solution for Pseudoephedrine, Ambroxol and Desloratidine respectively and $10 \mu \mathrm{l}$ solutions were injected into the system and the chromatograms were recorded to assess the stability of sample.

\section{Alkali Degradation Studies:}

To $1 \mathrm{ml}$ of stock solution of Pseudoephedrine, Ambroxol and Desloratidine, $1 \mathrm{ml}$ of $2 \mathrm{~N}$ sodium hydroxide was added and refluxed for $30 \mathrm{mins}$ at www.ijapbjournal.com $60^{\circ} \mathrm{C}$. The resultant solution was diluted to obtain $60 \mu \mathrm{g} / \mathrm{ml}, 120 \mu \mathrm{g} / \mathrm{ml}$ and $10 \mu \mathrm{g} / \mathrm{ml}$ solution for Pseudoephedrine, Ambroxol and Desloratidine respectively and $10 \mu \mathrm{l}$ solutions were injected into the system and the chromatograms were recorded to assess the stability of sample.

\section{Dry Heat Degradation Studies:}

The standard drug solution was placed in oven at $105^{\circ} \mathrm{C}$ for $6 \mathrm{hr}$ to study dry heat degradation. For HPLC study, the resultant solution was diluted to obtain $60 \mu \mathrm{g} / \mathrm{ml}, 120$ $\mu \mathrm{g} / \mathrm{ml}$ and $10 \mu \mathrm{g} / \mathrm{ml}$ solutions for Pseudoephedrine, Ambroxol and Desloratidine respectively and $10 \mu \mathrm{l}$ were injected into the system and the chromatograms were recorded to assess the stability of the sample.

\section{Photo Stability Studies:}

The photochemical stability of the drug was also studied by exposing the $0.1 \mathrm{mg} / \mathrm{ml}, 1.2$ $\mathrm{mg} / \mathrm{ml}$ and $0.1 \mathrm{mg} / \mathrm{ml}$ solution to UV Light by keeping the beaker in UV Chamber for 7days or 200 Watt hours $/ \mathrm{m}^{2}$ in photo stability chamber. For HPLC study, the resultant solution was diluted to obtain $60 \mu \mathrm{g} / \mathrm{ml}, 120 \mu \mathrm{g} / \mathrm{ml}$ and 10 $\mu \mathrm{g} / \mathrm{ml}$ solutions for Pseudoephedrine, Ambroxol and Desloratidine respectively and $10 \mu \mathrm{l}$ were injected into the system and the 
chromatograms were recorded to assess the stability of sample.

\section{Neutral Degradation Studies:}

Stress testing under neutral conditions was studied by refluxing the drug in water for 6 hrs at a temperature of $60^{\circ} \mathrm{C}$. For HPLC study, the resultant solution was diluted to obtain 60 $\mu \mathrm{g} / \mathrm{ml}, 120 \mu \mathrm{g} / \mathrm{ml}$ and $10 \mu \mathrm{g} / \mathrm{ml}$ solutions for Pseudoephedrine, Ambroxol and Desloratidine respectively and $10 \mu \mathrm{l}$ were injected into the system and the chromatograms were recorded to assess the stability of the sample.

\section{RESULTS AND DISCUSSION:}

The main aim for development of chromatographic method was to get reliable method for quantification of Pseudoephedrine, Ambroxol and Desloratidine from bulk and pharmaceutical dosage form and which will be applicable for the degradation products also. Different chromatographic conditions were employed for the analysis of the Pseudoephedrine, Ambroxol and Desloratidine in both bulk and pharmaceutical dosage form. Finally the analysis was performed by using Phosphate Buffer: Acetonitrile: Methanol in the ratio of $50: 20: 30 \% \mathrm{v} / \mathrm{v}$ at a flow rate 1.0 $\mathrm{ml} / \mathrm{min}$. Samples were analyzed at $225 \mathrm{~nm}$ at an injection volume of $10 \mu \mathrm{L}$ and separation was carried by using Inertisil ODS 3V, (250 x $4.6 \mathrm{~mm}, 5 \mu$ ), column. The retention time and tailing factor were calculated. The retention time of Pseudoephedrine, Ambroxol and Desloratidine were found to be $2.375,3.985$ and 5.458 respectively. The proposed column was selected which gave a sharp and symmetrical peak with 1.37 tailing factor and theoretical plates of 3120 for Pseudoephedrine, 1.23 tailing factor and theoretical plates of 4016 for Ambroxol and 1.45 tailing factor and theoretical plates of 3651 for Desloratidine.

The calibration curve was linear over the concentration range of 2.5-15 (ppm) (Pseudoephedrine), 30-180 (ppm) (Ambroxol) and 2.5-15 (ppm) (Desloratidine). Six different concentrations of Pseudoephedrine, Ambroxol and Desloratidine in the given range were prepared and injected into HPLC. The linearity of the method was statistically confirmed. RSD values for accuracy and precision studies obtained were less than $2 \%$ which revealed that developed method was accurate and precise. The system suitability parameters were given in table-6.

Forced degradation studies concluded that the all the degradant peaks obtained during degradation were well resolved from the main drugs i.e. Pseudoephedrine, Ambroxol and Desloratidine. And the peak purity was passed i.e. purity angle was less than purity threshold as per Empower-2 software. Hence the method is found to be stability indicating. The slope (m) and intercept (c) obtained were shown in the table- 6 .

Therefore proposed developed and validated stability indicating method was successfully applied to determine Pseudoephedrine, Ambroxol and Desloratidine in bulk and pharmaceutical dosage form.

\section{CONCLUSION:}

The developed method is accurate, simple, rapid and selective and proved to be stability indicating for the simultaneous estimation of Pseudoephedrine, Ambroxol and Desloratidine in bulk and pharmaceutical dosage form. The sample preparation is simple, the analysis time is short and the elution is by isocratic method. The retention time of Pseudoephedrine, Ambroxol and Desloratidine were found to be $2.375,3.985$ and 5.485 min respectively. The excipients of the commercial sample analyzed did not interfere in the analysis, which proved the specificity of the method for these drugs. Forced degradation studies of different conditions shows that all the degradants were well resolved from these main drug peaks and able to quantify the Pseudoephedrine, Ambroxol and Desloratidine in presence of degradants and excipients which proved that the method is found to be stability indicating. Hence the proposed method can be conveniently adopted for the routine quality control analysis in the bulk and combined pharmaceutical formulations.

\section{ACKNOWLEDGEMENT:}

The authors gratefully acknowledge Spectrum Pharma Research Solutions, Hyderabad, India 
for providing necessary facilities to carry out this research work.

\section{REFERENCES:}

1. Nada $S$, Abdelwahab and Eglal A. Abdelaleem. Stability indicating RP-HPLC method for simultaneous determination of guaifenesin and pseudoephedrine hydrochloride in the presence of syrup excipients. Arabian Journal of Chemistry, 2013.

2. Hussen Al-Akraa, Nazira Sarkis and Mohannad Alshehaby. New Rapid RPHPLC Method for Simultaneous Determination of Some Decongestants and Cough-Sedatives. International Journal of Pharmacy and Pharmaceutical Sciences, 2013; 5(4).

3. Nirav C Patel, Dipti B Patel and Pruthviraj K Chaudhari. Development and Validation of Reverse Phase High Performance Liquid Chromatographic Method for Simultaneous Estimation of Ambroxol Hydrochloride, Guaifenesin and Levosalbutamol Sulphate in Syrup. Inventi Rapid: Pharm Analysis \& Quality Assurance, 2013; (2).

4. Ekta Sharma, Nehal J Shah, Development and Validation of First order derivative Spectrophotometric Method for simultaneous estimation of Ambroxol Hydrochloride and Desloratadine Hydrochloride in Combined Tablet Dosage Form, International Journal of Pharmaceutical Research and Bio Science, 2012; 1(2): 155-166.

5. Sharma Ekta A and Shah Nehal J. Development and Validation of High Performance Thin Layer Chromatography Method for Simultaneous Estimation of Ambroxol Hydrochloride and Desloratadine Hydrochloride in their combined tablet dosage form. International Research Journal of Pharmacy, 2012; 3(5).

6. P.L.Sateesh, V.Pavithra, Bishupada biswal and G.Nagarjuna Reddy. Method development and Validation of Ambroxol Hydrochloride and Loratadine by RPHPLC in Tablet Dosage Form. International Journal of Pharma Sciences, 2013; 3(5): 370-374.
7. Krishna Veni Nagappan, Meyyanathan SN, Rajinikanth B Raja, Suresh Reddy, Jeyaprakash MR, Arunadevi S Birajdar and Suresh Bhojraj. A RP-HPLC Method for Simultaneous Estimation of Ambroxol Hydrochloride and Loratidine in Pharmaceutical Formulation. Research Journal of Pharmacy and Technology, 2008; 1(4).

8. Indian Pharmacopeia, Government of India, Ministry of health and family welfare, 2007, volume-II, published by Indian Pharmacopeia Commission, Ghaziabad, page no.701-702.

9. British Pharmacopoeia, Government British Pharmacopoeial Commission, 2011, volume I and II, page no. 265 (monograph 1489).

10. Prince Francis Moses, Prathap Singh, Alagar Raja and David Banji. Analytical Method Development and Validation for Simultaneous Estimation of Ambroxol and Desloratadine in its Pharmaceutical Dosage Form by RP-HPLC. World Journal of Pharmacy and Pharmaceutical Sciences, 2013; 2(6): 6246-6262.

11. G. Babu, Shirin K and R. Rajapandi. RPHPLC method for the simultaneous estimation of Ambroxol hydrochloride and Desloratadine in pure and dosage form. Der Pharmacia Lettre, 2013; 5(3): 391-396.

12. Rele Rajan V and Gurav Pankaj J. Simple Spectrophotometric Methods for Determination of Ambroxol Hydrochloride from Pharmaceutical Formulation. International Journal of PharmaTech Research, 2012, 4(3): 994998.

13. Krupa M. Kothekar, Balasundaram Jayakar, Amit P. Khandar and Rajnish K. Mishra, Quantitative Determination of Levofloxacin and Ambroxol hydrochloride in Pharmaceutical Dosage Form by Reversed Phase High Performance Liquid Chromatography. Eurasian Journal of Analytical Chemistry, 2007, 2(1): 21-31.

14. Jigar Goswami, Jagdish Kakadiya and Nehal Shah. Development and Validation of First Order Derivative Spectrophotometric Method for Simultaneous Estimation of Ambroxol and Cefpodoxime in Combined Tablet 
Dosage Form. International Journal of Pharmaceutical and Chemical Sciences, 2012; 1(2).

15. K.B. Shalini, Nalini Kanta Sahoo, B. Gopinadh, T. Jyothi1 and P. Vijay Kumar. Development and Validation of Loratadine in Bulk and Pharmaceutical Dosage Form by UV Spectroscopic Method. International Journal of Pharmaceutical Research and Analysis, 2014; 4(1): 39-43.

16. J. Valarmathy, L. Samuel Joshua, N.T.H. Guptha, M.Ganesh, A. Lakshmana Rao and T. Siva Kumar. RP-HPLC Estimation of Desloratadine in Pharmaceutical Dosage Form. Asian Journal of Chemistry, 2009; 21(9): 7431-7433.

17. Dina T. El-Sherbiny, Nahed El-Enany, Fathall F. Belal and Steen H. Hansen. Simultaneous
Loratadine and Desloratadine in pharmaceutical preparations using liquid chromatography with a microemulsion as eluent. Journal of Pharmaceutical and biomedical analysis, 2007; 43: 1236-1242.

18. Rele Rajan. V and Gurav Pankaj J. A simple Extract active Spectrophotometric Determination of Loratadine, Desloratadine and Rupatadine from Pharmaceutical Formulations. International Journal of Pharma and Biosciences, 2012; 3(2): 89.

19. The Merck Index, published by Merck \& Co., Inc., Whitehouse station, $14^{\text {th }}$ ed., NJ, USA, page no. 385.

20. Martindale, the complete drug reference, $34^{\text {th }}$ ed., published by Pharmaceutical press, page no. 1114.

21. Validation of Analytical Procedures: Text and methodology-ICH Q2-R1. 